\title{
BIOPHYSICAL STIMULATION OF BONE FRACTURE REPAIR, REGENERATION AND REMODELLING
}

\author{
Edmund Y.S. Chao* and Nozomu Inoue \\ Biomechanics Laboratory, Department of Orthopaedic Surgery \\ Johns Hopkins University, School of Medicine, Baltimore, Maryland 21205-2196
}

\begin{abstract}
Biophysical stimulation to enhance bone fracture repair and bone regenerate maturation to restore its structural strength must rely on both the biological and biomechanical principle according to the local tissue environment and the type of mechanical stress to be born by the skeletal joint system. This paper reviews the possible interactions between biophysical stimuli and cellular responses in healing bone fractures and proceeds to speculate the prospects and limitations of different experimental models in evaluating and optimising such non-invasive interventions. It is important to realize that bone fracture repair has several pathways with various combinations of bone formation mechanisms, but there may only be one bone remodeling principle regulated by the hypothesis proposed by Wolff. There are different mechanical and biophysical stimuli that could provide effective augmentation of fracture healing and bone regenerate maturation. The key requirements of establishing these positive interactions are to define the precise cellular response to the stimulation signal in an in vitro environment and to use well-established animal models to quantify and optimise the therapeutic regimen in a time-dependent manner. This can only be achieved through research collaboration among different disciplines using scientific methodologies. In addition, the specific forms of biophysical stimulation and its dose effect and application timing must be carefully determined and validated. Technological advances in achieving focalized stimulus delivery with adjustable signal type and intensity, in the ability to monitor healing callus mechanical property non-invasively, and in the establishment of a robust knowledgebase to develop effective and reliable treatment protocols are the essential pre-requisites to make biophysical stimulation acceptable in the main arena of health care. Finally, it is important to bear in mind that successful fracture repair or bone regeneration through callus distraction without adequate remodeling process through physiological loading would seriously undermine the value of biophysical stimulation in meeting the biomechanical demand of a long bone.
\end{abstract}

Key Words: Biophysical stimuli, fracture repair, bone regenerate, ultrasound, pulsed electromagnetic field, shock wave.

*Address for correspondence:

E.Y.S. Chao

Biomechanics Laboratory,

Department of Orthopaedic Surgery

Room 235, Ross Building, JHU School of Medicine

720 Rutland Ave, Baltimore, MD 21205-2197

Telephone Number: 4105026416

FAX Number: 4105026414

E-mail: echao@jhmi.edu

\section{Introduction}

It is well known that a bone fracture will repair and remodel depending on the ensuing loading conditions (Wolff, 1986; O’Sullivan et al., 1989; Meadows et al., 1990; Aro and Chao, 1991; Cowin, 1993). Both cortical and cancellous bone morphology have been related to the structural stress pattern based on theoretical analysis (Hart et al., 1984; Rubin and Lanyon, 1984, 1987; Carter, 1987; Hart, 1990). It is very likely that during bone repair and regeneration, the type of stress applied may dictate its material and structural quality. In musculoskeletal system, the biomechanical environment plays a key role in repairing, maintaining, and remodeling of bone in meet its functional demands. Based on this fundamental concept, many connective tissue remodeling rules have been proposed to explain the repair process and their biological responses (Chow et al., 1987). When the normal healing and remodeling environment is absent or compromised, reliable and effective biological or biophysical stimulation may be indicated.

Unfortunately, many of the basic biological and biomechanical interactions affecting different connective tissue response are poorly understood. Without this knowledge, it would be difficult to identify the specific cell mediating mechanisms that regulate the normal or delayed repair after bone fracture. Such biologic and biomechanical interactions can help us to identify abnormal repair processes and uncover the enhancing factors for the purpose of augmenting bone fracture healing or bone regenerate maturation. Therefore, it has been the goal of many investigators to search for the relationship between biophysical factors and cellular responses under normal and deficient bone fracture healing conditions. To establish the interdependence of biophysical stimulation and bone repair and remodeling at the material and structural level, experiments must be carefully designed and performed using appropriate animal models to investigate these cellular and tissue responses under different forms of biophysical stimulation. When necessary, in vitro cell and tissue culture studies under well-controlled biophysical stimuli must be conducted in order to isolate other confounding factors at the systemic level. Without knowing the normal histomorphometric and cellular responses associated with different bone fracture healing processes in quantitative terms, it would be nearly impossible to investigate potential stimuli to establish their efficacy in enhancing such a complex biological process.

In any form of fracture fixation, bone fragments under load will experience certain amount of relative motion, which, by unknown mechanisms, determines the morphologic features of fracture repair. Perren (1979) proposed a brilliant hypothesis, the "Interfragmentary Strain Theory", which related the tissue response to the local 

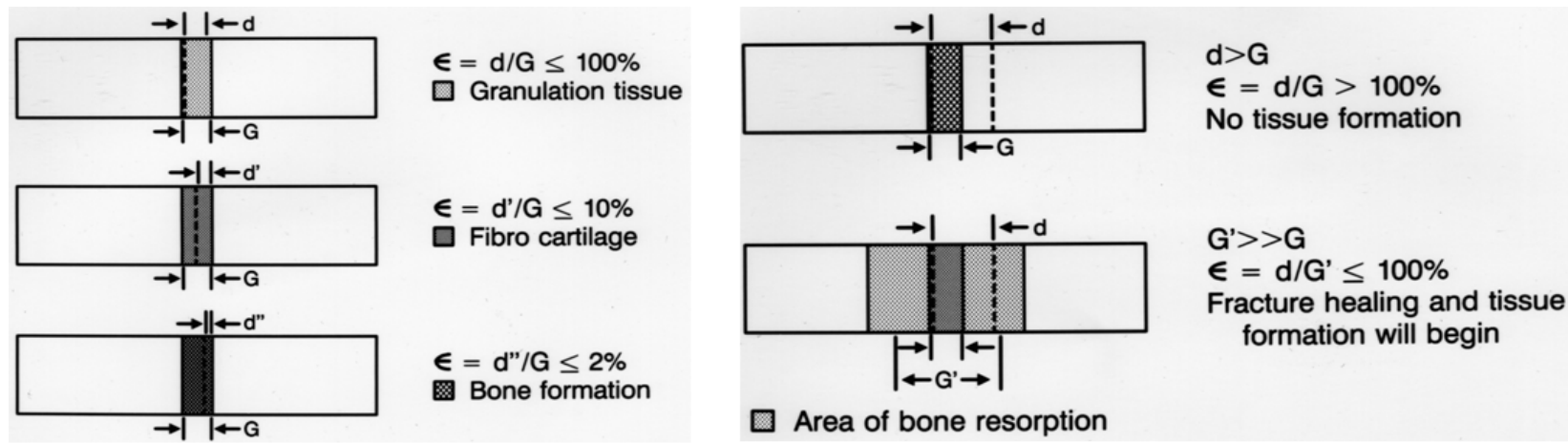

Figure 1. The one-dimensional Interfragmentary Strain Theory of Perren (1979) G= gap size; d, d', d" = fracture gap motion; e = interfragmental strain. LEFT: The transformation of tissue type based on normal strain at the fracture gap when fracture gap motion is smaller than the gap size. RIGHT: The transformation of tissue type in the gap when gap motion is larger than the gap distance. In this case, the bone near the gap will be resorbed and thus making the gap distance so that the interfragmental strain will reduce to $<100 \%$. Unfortunately, when the gap becomes too large, non-union or delayed-union may occur even with adequate fixation method and immobilization.

mechanical environment. The interfragmentary strain was defined as the ratio of the relative displacement of fracture ends versus the initial gap width (Fig. 1). Although such a concept was an oversimplification of the biomechanical response of the opposing bone and gap tissue, the underlying phenomenon had successfully demonstrated the governing principle of mechanical intervention of tissue formation and transformation. The fracture gap tissue and the existing bone cortex remodeling appeared to follow this rule to prepare for solid bone union. However, the timerelated changes in the external callus versus its local deformation under assumed loading conditions did not seem to fit the interfragmentary strain theory (Augat et al., 1998).

The original interfragmentary strain theory considered only longitudinal strain along the axial direction (Perren, 1979). Analytical three-dimensional analysis (Cheal et al., 1991) revealed a complex gap deformation and multidirectional principal strains not even considering the extramedullary and intramedullary callus. There may also be additional regulating mechanisms for tissue differentiation during initial fracture healing (Carter et al., 1988). Therefore, the interfragmentary theory had its limitations although it was intended to conceptualize the mechanism involved in achieving "contact healing" or "gap healing" without periosteal callus. A more general concept would be necessary to deal with the biomechanical effects on fracture repair under a new classification system (Aro and Chao, 1993) based on histological appearance of the healing tissue around the fracture site under different fixation methods. This knowledge can guide us to explore additional biophysical stimuli to modulate bone union pathways. The main objective of this review paper was to discuss the logic, the past research, future prospects, and the potential pitfalls associated with the development of more appropriate models to clarify the relationship between biological relations and bone fracture healing enhancement using different biophysical stimulation modalities. It is hoped that the knowledge to be gained could also be expanded to the field of limb lengthening through callus distraction and bone quality maintenance under osteoporosis.

\section{Physical Modulation of Bone Fracture Healing and Remodeling}

It has long been known that mechanical stimulation can induce fracture healing or alter its biological pathway (Rand et al, 1981; Brighton, 1984; Wu et al., 1984; Aro and Chao, 1991; Claes et al, 1997). Repetitive loading under small strain and high frequency or overloading through elevated exercise regime has been demonstrated to cause bone hypertrophy (Goodship and Kenwright, 1985; Rubin et al., 2001). The added bone formation is also related to the direction and magnitude of overloading which will affect the internal state of stress of the repairing tissue. However, the regulating cellular mediators responsible for such a phenomenon remain unknown (Fig. 2). If the underlying effect at the cell membrane or cytoplasmic level could be directly linked to the biophysical stimulant, effective and reliable method to maintain or enhance bone regeneration may be established for the treatment of difficult fractures in patients with deficient osteogenic potential due to either local or systemic abnormalities.

When the mechanisms for tissue formation at the cellular level are understood and well defined, physiological conditions or pharmacological agents may be developed

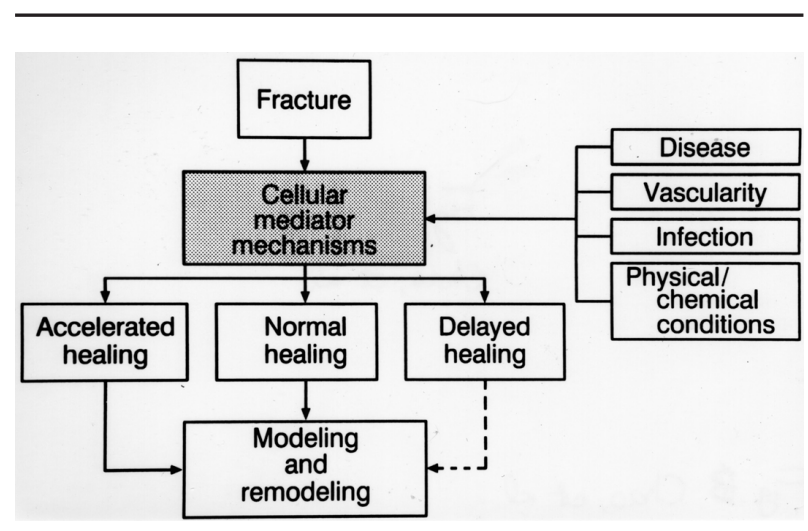

Figure 2. Regulatory pathway for bone fracture repair and remodeling with and without biologic or physical stimuli. 

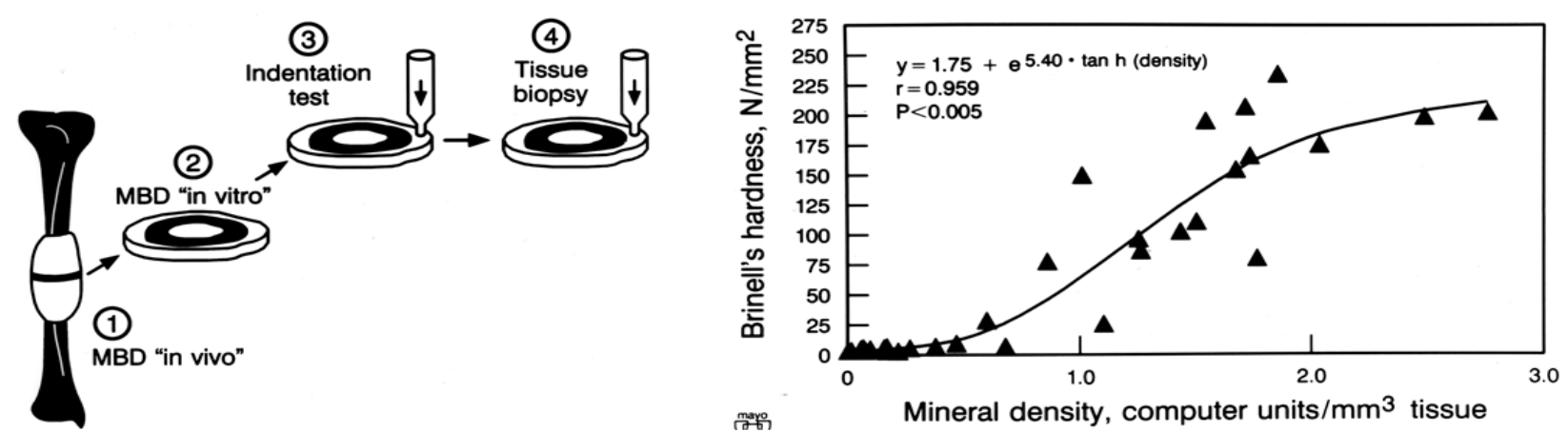

Figure 3. Relationship between the indentation hardness of the fracture gap tissue and periosteal callus and their mineral density expressed in the computer units according to the gray level calibrated using a stepped wedge scale. (a) Callus tissue under indentation test after bone mineral density measurement with subsequent tissue biopsy for ash content analysis. (b) The non-linear regression relationship between indentation hardness values and the equivalent bone mineral density measurement throughout the fracture healing process.

to accomplish the same callus formation and bone regeneration effects without the mechanical interventions which are often difficult to administrate under adverse conditions. However, the potential mechanoreceptors on the cell membrane sensitive to stress/strain induced by electromechanical or stream potential signals have yet to be identified. Such a discovery, if successfully accomplished, can significantly help to unravel the mystery of regulating pathway for connective tissue remodeling and disuse atrophy, which has only been theorized without validation. Before this is accomplished, the clinicians treating bone fractures must understand that there are different biological, physiological, and mechanical factors which can have either positive or negative effects on fracture repair at the tissue level. It is equally important to recognize the possibility that mechanical loading may be the only irreplaceable element in governing bone remodeling following successful initiation of the fracture repair process.

\section{Surgical intervention and biomechanical environment on fracture repair}

Both rats and canines have been used as the experimental animal models for manually created long-bone fractures or surgically produced transverse and oblique osteotomies (Aro et al., 1989; Aro et al., 1990b; Aro et al., 1991). Gap and contact with and without static compression were used as the fracture end conditions, while rods, compression plates, and external fixators of different stiffness properties were utilized as the means of studying the effects of several fracture immobilization methods on bone healing patterns. In addition, passive axial dynamization through relaxing the telescoping mechanism on the side bar of an external fixator was also used to study its possible effects of fracture site compression on fracture callus and bone histomorphometry (Aro et al., 1990a). Roentgenographic, nuclear scintigraphic, static and dynamic weight bearing, histological, biomechanical, cellular, and biochemical methods were used to quantitate the histomorphometry, mineral density, and mechanical properties of callus and newly formed bone in order to identify the unique features associated with each fracture type and fixation condition. The data associated with different experimental conditions was used to characterize different fracture healing types previously identified or yet unrecognized.

Under rigid internal or external fixation, fracture union morphology matched that of the contact or primary healing mechanism, with direct osteonal migration across the fracture gap. However, both periosteal and endosteal callus and new bone formation were common, depending upon the loading condition and micro-movement at the fracture site inherent to the specific immobilization method used. Interfragmentary compression did not alter the basic morphologic features of fracture repair, except for the proportional reduction of periosteal callus. Osteonal migration also occurred despite the presence of fracture gap, although the woven bone within the gap had transversely oriented collagen fibers laced with longitudinal traversing osteons. When less rigid internal fixation (intramedullary nail without interlock) and external fixation (smaller and fewer pins in unilateral frame) methods were employed, fracture repair followed the secondary healing mechanism with an abundant amount of periosteal and endosteal callus but without osteonal migration (Rand et al., 1981). The distribution of endochondral ossification and intramembranous ossification depended upon the biologic and biomechanical environment. The transformation from callus to mineralized woven bone occurred early in the healing period and increased its volume to replace cartilage and undifferentiated tissue (Markel et al., 1990). This transformation was responsible for the mechanical strength or the cortical bone as demonstrated by the mechanical indentation results in different regions of the maturing callus in a fracture-healing model (Fig. 3).

Axial dynamization, both passively and actively, under external fixation in stable and unstable fracture types provided an increased amount and more uniform distribution of periosteal callus (Aro et al., 1990a). As the load was transmitted through the fracture site after dynamization, there were fewer pin tract problems due to reduced pin/bone interface stress. However, changing fixator stiffness by removal of excessive pins or connecting sidebars did not show any positive effect on augmenting bone fracture healing. Without exception, weight bear- 
ing was proven to be important, especially in restoring the fractured bone to its original mechanical strength. Finally, axial dynamization was able to enhance more advanced bone remodeling in distraction osteogenesis which is closely related to the loading response according to the Wolff's hypothesis (Windhager et al., 1995).

\section{Effects of physical loading on fracture healing pathways}

To better describe the effects of mechanical and biological influences on bone fracture repair and remodeling, a revised classification of bone union mechanisms (Chao and Aro, 1989) was proposed to replace the oversimplified primary and secondary bone healing types. The new classification was derived from the histological evaluation on the absence or presence of secondary osteons across the fracture gap. Periosteal callus could co-exist with osteon migration when the mechanical environment at the fracture site was appropriate. During fracture repair, there were four basic new bone formation processes: 1) osteochondral ossification, 2) intramembranous ossification, 3) oppositional new bone formation, and 4) osteonal migration (creeping substitution). Bone regenerate through callus distraction is a combination of these basic bone formation processes. The type of bone formation processes and their occurrence would vary according to many factors related to fracture type, gap condition, fixation rigidity, loading and biologic environment. Regardless of the fracturehealing pathway, mechanical intervention might be the only means to assure bone remodeling after successful callus formation and maturation in order to restore the bone to its original structure and strength. It was proposed that the same bone augmentation effect could also regulate bone remodeling by establishing the optimal strain threshold (Fig. 4) as well as loading frequency but such a contention remained to be validated especially in the light of the recent interest of low bone strain as an anabolic stimulus for bone quality maintenance (Rubin et al., 2001).

\section{Basic Forms of Biophysical Stimuli on Bone Fracture Healing}

As early as 1955, Yasuda had already discovered the Electric Callus phenomena and postulated that "dynamic energy exerted upon bones is transformed into callus formation" (Yasuda, 1955). Now after nearly a half century, the ability to manipulate bone and other connective tissue using external energy is still doubted by some in spite of years of basic research and clinical investigations. Instruments delivering low intensity pulsed ultrasound (LIPU) (Heckman et al., 1994; Yang et al., 1996; Bolander, 1998), pulsed electromagnetic fields (PEMF) (Bassett, 1961; Sharrard, 1990; Eyres et al., 1996; Ryaby, 1998), low power direct current (DC) (Brighton et al., 1981; Brighton and Hunt, 1986), extracorporeal shock wave stimulation (ECSW) (Schaden et al., 2001; Wang et al., 2001), and the low intensity high frequency vibration (LIHFV) (Rubin et al., 2002; Srinivasan et al., 2002; Tanaka et al., 2003) are being promoted by the medical instrument industry with mixed responses in the orthopaedic community. These are the basic forms of biophysical stimuli but it is still contro-

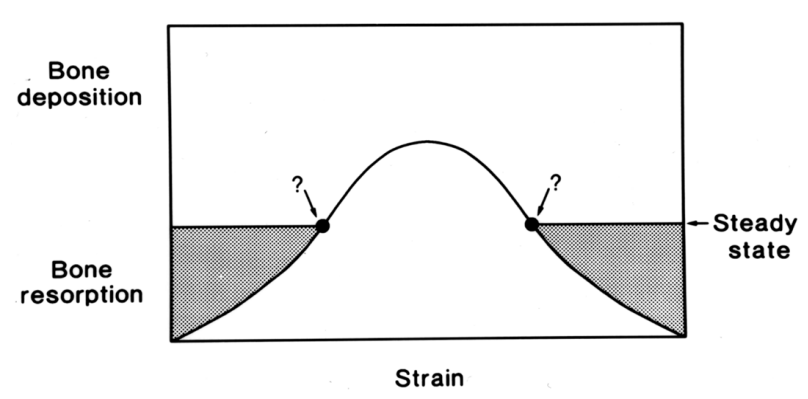

Figure 4. The unknown strain threshold to be established for bone formation or resorption. Such hypothetical bone strain range, if exists, would probably vary according to the type of bone involved and its healing or degeneration stages.

versial whether these modalities produce different cellular responses or they all follow a similar osteogenic pathway. The importance of utilizing well-established in vitro tissue culture models must be emphasized to supplement the cellular study results for biophysical stimuli signal and dose effect optimisation.

\section{Biological response}

Cultured cell and tissue subjected to different physical and electrical signals of varying intensity in an in vitro setting have been studied using the molecular biology and histomorphological analyses. Single cell under carefully controlled stimulation environment using specially designed equipment was conducted to investigate the basic mechanism of cellular response under stimulation (Bolander, 1998). Biochemical pathways activated in signal transduction under various types of electrical stimulation have also been investigated on bone cells (Brighton et al., 2001). Various animal models from rats, rabbits, canines, sheep, to horses simulating fresh fracture, delayed union, limb lengthening, etc. were studied to evaluate the energy sources and their dose effects on tissue response judging from the radiographic, histomorphological, and biomechanical results.

The low intensity pulsed ultrasound was found to enhance fracture healing by stimulating earlier synthesis of extracellular matrix protein, the aggregan in cartilage, possibly altering chondrocyte maturation through endochondral bone formation pathway (Yang et al., 1996). Pulsed electromagnetic fields stimulation was found to induce osteogenesis through upregulating BMP-2 and BMP-4 in osteoblasts (Bodamyali et al., 1998). The application of direct current reduced local tissue oxygen concentration which could transform polymorphic cells to bone (Brighton and Hunt, 1986). Such mechanism also applied to mesenchymal cells associated with bone fracture hematoma. It has been postulated that the extracorporeal shock waves caused microtrauma or microfracture and induce neovasculization through hematoma formation, which would increase osteoblast or fibroblast activity (Wang et al., 2001). Unfortunately, biological studies at cellular level cannot provide reliable information on the therapeutic effect of biophysical stimulation on tissue response at the system level. 

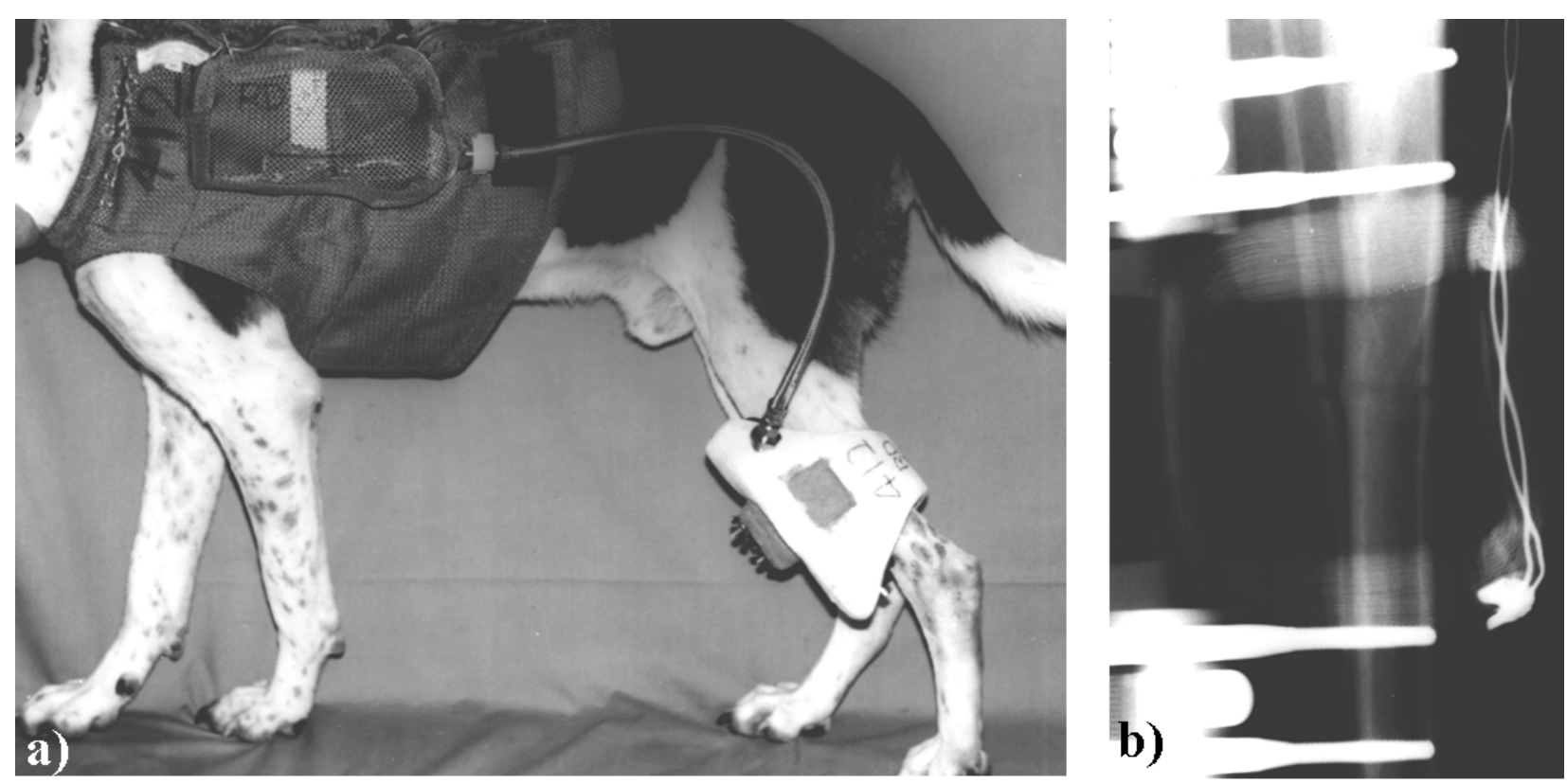

Figure 5. Effect of PEMF (Pulsed Electro-Magnetic Field) stimulation on delayed fracture union enhancement. (a) The canine mid-tibia osteotomy gap model with externally mounted coil protected by a plexiglass guard and the stimulator/battery backpeck. (b) The radiographic appearance of the stimulator coil centered at the osteotomy site with bone segments stabilized by an external fixator.

\section{Experimental results in animal models}

In animal experiments, positive effects on bone fracture healing enhancement were found consistently in different models and under a variety of biophysical stimuli. The type of tissue formation in the bone healing process was found to follow closely the cellular mechanism associated with the specific form of energy. In a well-controlled canine unilateral delayed union model, pulsed electromagnetic fields (PEMF) stimulation for one hour per day for a total of eight weeks significantly increased weightbearing on the affected limb with higher mechanical strength of the healing osteotomy due to increased periosteal new bone formation (Fig. 5). Another striking finding in this study was the effect of pulsed electromagnetic fields on the reduction of cortical porosity in the bone adjacent to the osteotomy when compared to the non-treated group (Fig 6) (Inoue et al., 2002). However, in a rabbit tibial lengthening model, neither LIPU nor PEMF using the current signal type and dose had demonstrated any significant enhancement effect on bone regenerate maturation (Tis et al., 2002; Taylor et al., 2003).

\section{Clinical use of biophysical stimulation}

There are numerous clinical reports to support effectiveness of biophysical stimulation on fresh fracture, delayed union, and bone lengthening. Several prospective, randomized clinical studies have shown the efficacy of low intensity pulsed ultrasound (LIPU) in stimulating bone formation after fracture (Heckman et al., 1994, Kristiansen et al., 1997), non-union (Xavier and Duarte, 1983), and bone
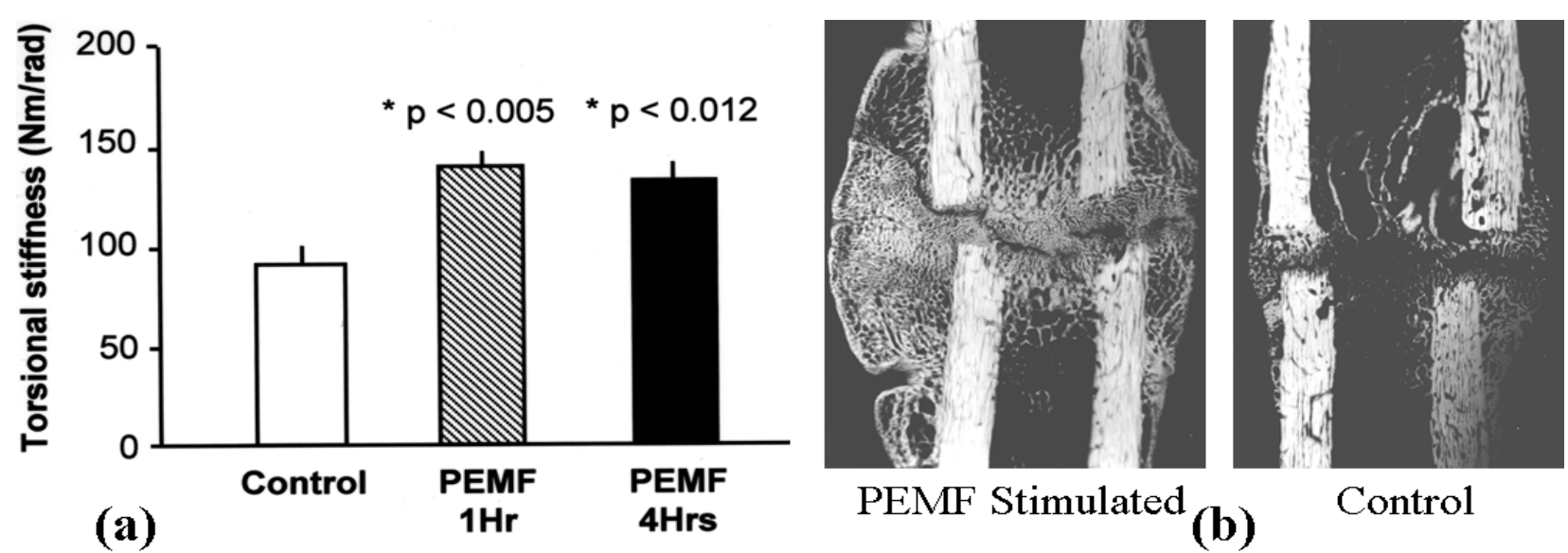

Figure 6. (a) Effects of PEMF with two dose regimens (1 and 4 hour stimulation) on bone structural stiffness under torsion in an osteotomy delayed union model when compared with the un-stimulated control group (data expressed in mean value and standard error of the mean). (b) Comparison of the microradiographic results on new bone formation at and around the osteotomy site between the PEMF stimulated side and the unstimulated control side. Please note the much-reduced porosity in the intact cortex on the stimulated side. 
lengthening (Sato et al., 1999). Pulsed electromagnetic fields (PEMF) stimulation has been in clinical use for nearly 30 years on patients with delayed fracture healing and nonunion and demonstrated its effect in a multitude of clinical case reports (Bassett, 1989; Eyres et al., 1996; Ryaby, 1998). Double-blinded studies confirmed the clinical effectiveness of pulsed electromagnetic fields stimulation on osteotomy healing (Borsalino et al., 1988; Mammi et al., 1993) and delayed union fractures (Sharrard, 1990). Brighton et al. (1981) conducted multi-center study of the nonunion and reported an $84 \%$ clinical healing rate of nonunion with direct current treatment. Recently, Schaden et al. (2001) reported $76 \%$ of non-union or delayed union patients treated with one time extracorporeal shock wave therapy resulted in bony consolidation with a simultaneous decrease in symptoms.

All these results would strongly support the efficacy of biophysical stimulation as a therapeutic modality in bone fracture repair and bone regenerate augmentation. However, current commercial devices need to utilize the available bioengineering technology to optimize instrument design and treatment protocol development to build biophysical stimulation on firmer foundation. Insufficient effort has devoted to quantify the dose effect by considering timerelated tissue change as a factor to adjust the stimulation signal intensity in the treatment protocol. Additional research is required to specify field intensity and signal scattering as a function of local tissue attenuation and application variation. The basic science knowledge related to connective tissue modulation through external energy stimulation must be transformed into the technology of implementation, which could then be brought to the clinical arena as a system under physician's supervision to provide reliable and cost-effective enhancement on tissue repair, regeneration, remodeling, and maintenance when indicated. A grand vision of Non-invasive Tissue Engineering using biophysical stimulation needs to be shared by the investigators and clinicians as the ultimate goal of such therapeutic modality.

\section{Future Effort Required in Biophysical Stimulation}

In vitro cell and tissue culture studies are needed to explore the existence of a cellular response to biophysical stimulants. Under such an experimental setup, the basic biological mechanism responsible for the stimulation effect as well as the potential pitfalls of such intervention could be established. However, the conditions provided for a single cell or a cell population in a culture dish could not reproduce the actual environment in living tissues subject to physical loading. The same limitation may also exist in isolated tissue experiments in a bioreactor. Therefore, useful feasibility studies prior to clinical trial must rely upon well-designed animal experiments using models from which the signal/dose effect on tissue augmentation in response to a biophysical stimulation could be firmly established and quantified.

\section{Animal model selection}

To establish bone fracture enhancement phenomenon, animals that can be studied under a simple but well-con- trolled environment with fast response, minimum species related variability, and low cost should be utilized. A large number of animals could then be used in order to perform time-sequenced studies so that the cellular events at different time periods can be captured to fully quantify the initiation, passage, maintenance and possible reversal of such an interaction. Various molecular biology techniques and DNA probes must also be available to describe the interaction at the molecular and cellular levels. In this type of experiment, it may not be critical to specify the intensity and distribution of the stimuli at the local tissue but data must be carefully stratified against that from the sham control group to rule out other confounding effects. Lower animal species, such as rats and rabbits, would be ideal for such purpose.

For definitive study at the tissue and structural level to establish optimal dose, clinical indications and treatment protocol, large animals with similar loading and physiological conditions to that of humans should be applied. The state of stress and strain of the local tissues will be determined through theoretical modeling under measurable external loading conditions. The reactive tissues in the model should be allowed to undergo remodeling subjected to varying biomechanical loading and tissue histomorphological changes as a function of time. Such studies, if properly executed, will provide the essential data to isolate the effects of biophysical stimulation effects on tissue repair, formation, remodeling and maturation from the normal responses. Experiments of this nature will provide the validity of biophysical stimulation needed to rationalize its therapeutic efficacy in clinical patient care with the necessary knowledgebase and technical information to stage clinical trial to assure treatment outcome quality assurance.

\section{Mechanical manipulation in bone regenerate}

Connective tissue manipulation is an important orthopedic procedure in treating limb length discrepancy and angular malformation. Such operative intervention has also been used in the treatment of fracture non-unions (Ilizarov, 1989; Delloye et al., 1990; Marsh et al., 1992). The most commonly applied technique involves callus distraction. To effectively achieve such mechanical manipulation, a waiting period after bone osteotomy is mandatory. The length of this crucial time period varies and there is no objective guideline to estimate it in each case using available information related to tissue compliance and cellular response (Yasui et al., 1993). In addition, the rate of distraction remains controversial. It would be desirable if the rate of bone distraction could be determined using a noninvasive method of assessing bone regenerate's biomechanical strength thereby implement the optimal remodeling conditions for bone (Windhager et al., 1995; Kassis et al., 1996). Research pertaining to the interaction of biomechanical forces and cellular responses will help to establish these essential guidelines. Such experimental studies can also provide useful information on applying proper compressive loading to enhance the consolidation of the distracted tissues. Similar investigations are expected to produce valuable information concerning the distractibility of vessels and nerves in the same operation. It has been proposed that 
the type of stress applied to immature or undifferentiated tissue, can dictate its regeneration fate (Carter et al., 1988) (Fig. 7). If these connective tissue manipulation mechanisms are well established, they can be utilized through appropriate biophysical stimulation to form the required knowledgebase for the ultimate goal of Non-invasive Tissue Engineering.

\section{Experimental bone fracture model for biophysical stimulation study}

Attempts have been made to relate loading conditions to tissue formation during fracture repair. These investigations were focused on relating the strain levels within the hematoma and fracture callus which develop in the early stages of fracture healing to the type of tissue that forms. In general, areas of high strain and hydrostatic pressure within the repair tissue would relate to formation of cartilaginous tissue, while areas of low strain would induce the formation of bone (Cowin, 1993; Huiskes and Hollister, 1993; Claes and Heigle, 1996; Kuiper et al., 1996; Claes et al., 1997). In fracture models, high strain within the repair tissue could be interpreted as micromovement between bone ends at the fracture site, which might inhibit bone formation. A predictive model was developed to speculate the pattern of fracture repair based on "fuzzy" logic (Ament and Hofer, 2000). Bone, cartilage, fibrous and granulation tissue were modeled using a finite element analysis, with a set of empirically determined "fuzzy rules" driving the healing process based on the strain energy density and a vascularization factor. In these models, significant geometric and material property simplifications were used to model the fracture repair process and only axial loads were considered. These assumptions plus the empirical rules proposed for bone healing regulation making such model difficult to study the effect and mechanism of biophysical stimulation.

In addition, fracture healing is highly sensitive to the type of fixation devise employed, the local tissue environment, and the fracture gap condition under load. In most immobilization conditions, a combination of endochondral and intramembranous ossification processes are involved. If the fracture site had some potential to initiate callus dominant repairing mechanism, biophysical stimulation may have less consequence in producing significant enhancing effect. In bone lengthening or fracture under rigid fixation, however, a different mechanism governed predominantly by the intramembranous ossification pathway would be more suitable in studying the effects of biophysical stimulation.

\section{Cortical defect repair/remodeling model for biophysical stimulation study}

Unlike fracture repair, a cortical defect provides an environment in which loading increases the strain in the repair tissue and the surrounding bone without introducing micromotion. Hence, the repairing tissue would undertake an intramembranous ossification pathway (Fig. 8). The strain energy density within the intact cortical bone around the defect could be treated as a driving stimulus in the early repair stage to initiate a biological effect. This was the proposed working hypothesis on the regulating mechanism for

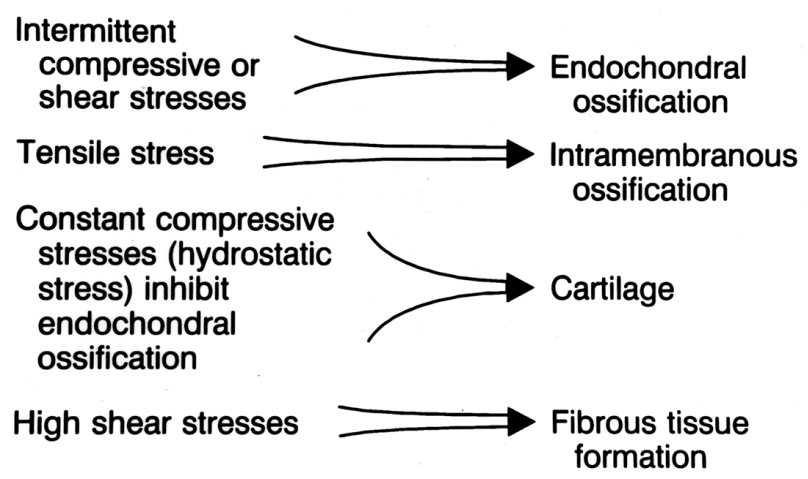

Figure 7. Hypothetical bone formation mechanism under different types of physical stresses.

cortical defect repair, a strain induced angiogenic process (Chao et al., 2004) The magnitude and orientation of the stress/strain distribution within the repair tissue may govern the modeling response by replacing the repair tissue with woven bone. Concerning remodelling in this unique situation, there might be a secondary mechanism regulated by minimizing the strain energy gradient while attempting to restore the long bone's structural strength for its functional demands. However, a scalar representation of the stress/strain field in terms of strain energy may not be appropriate for examining the modeling response in trabecular or woven bone, the magnitude and direction of the principal stresses within the defect would provide the regional influence on the material properties and orientation of the newly formed bone (Elias et al., 2000). It is therefore reasonable to postulate that the repair and remodeling processes would act to minimize strain energy density gradients within the system while the structural strength could be recovered for the loading requirement (Inoue et al., 2003). Therefore, cortical defect repair may never be complete morphologically even after a long period of normal activity (Claes et al., 1995). This model provides the ideal environment to study both biophysical and biological stimuli to improve bone repair and remodeling in the absence of a cartilaginous phase of bone formation.

The bone defect-healing model is simple with minimal risk of surgical variability and allows accurate stress/strain analysis. Immunohistochemistry and histomorphometry data would be sufficient to show the relationship between the loading conditions and the cellular response. Mechanical testing could be used to quantify the material properties of the immature and matured new bone. Once the repair and remodeling processes were established, additional studies may be conducted to investigate how temporal variations in mechanical intervention would influence the repair and remodeling processes. This model was applied to investigate the relationships among factors related to cell aging, decreased functional capacity, deficiency in tissue repair (Buckwalter et al., 1993), and to study the effects of pharmacological agents of (Gallagher et al., 1994; Majeska et al., 1994) or exercise programs (Eickhoff et al., 1993; Menkes et al., 1993) on osteoblastic and osteoclastic activity. It could also be used to elucidate the effects of BMPs and biophysical stimuli on bone regeneration through distraction which has similar histologic features as compared 


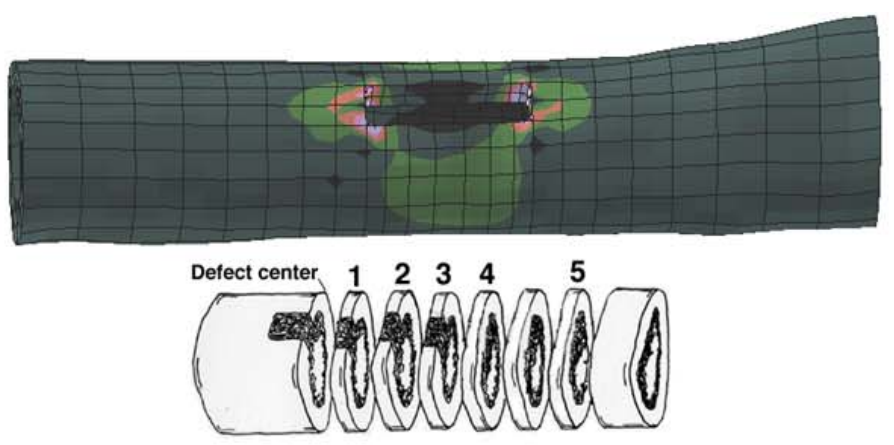

(a)
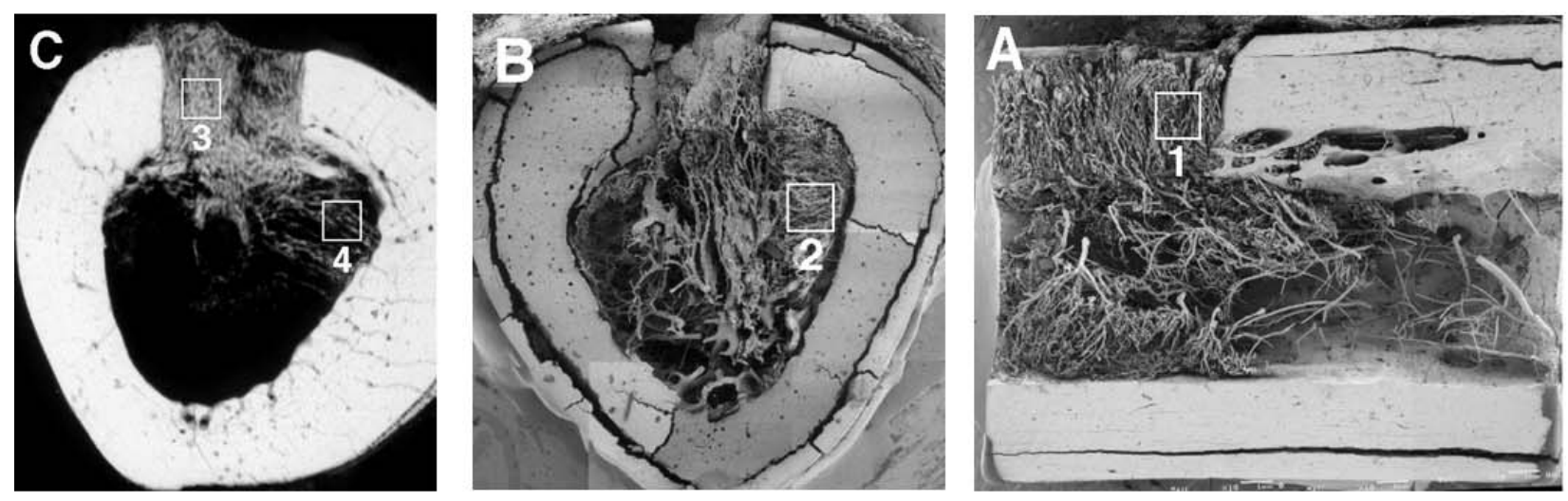

(b)

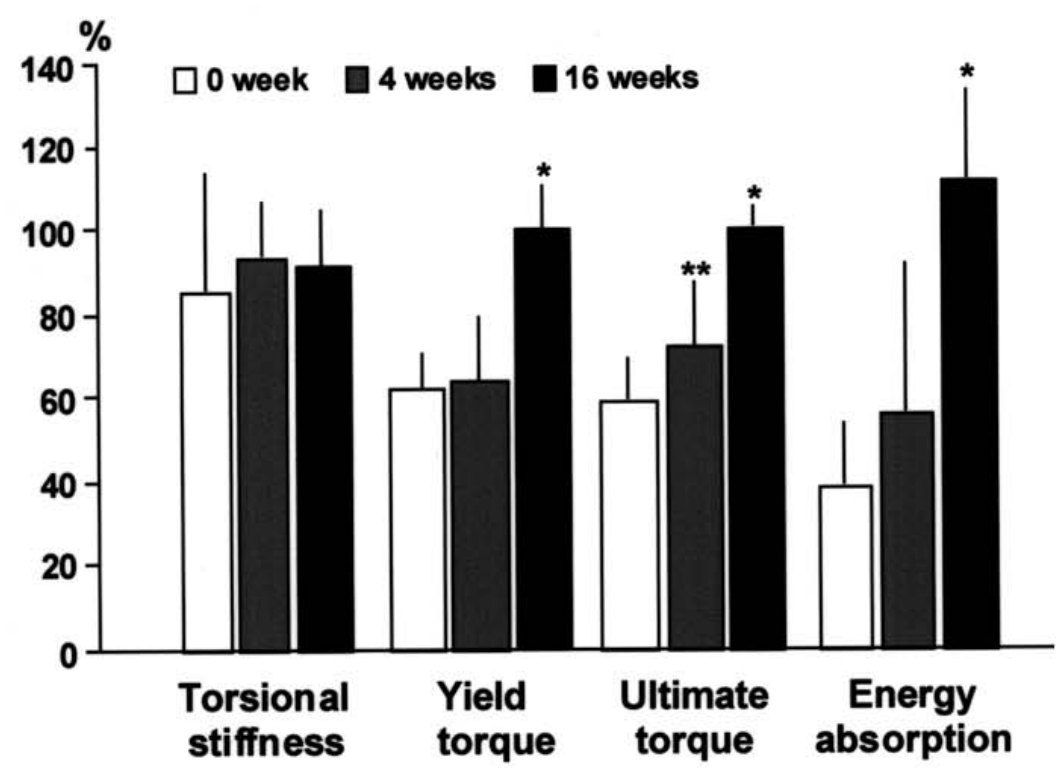

(c)

Figure 8. (a) Cortical defect repair model and the histomorphometric analysis location along and adjacent to the defect. (b) The microvascular network obtained using the plastic-investment technique (A: The longitudinal crosssection; and B: The transverse cross-section. Areas " 1 " and " 2 " were used for vascular density and orientation analysis) and the contact microradiograph (C: The transverse cross-section at the defect mid-section. Areas " 3 " and " 4 " were used to study trabecular bone density and architecture using Fourier Transform technique) of the bone at the defect 4 weeks after surgery. (c) The recovery of structural strength under torsion of the tibia containing the cortical defect as a function of healing time. 
to that in the cortical defect repair (Brighton et al., 1992; Cunningham et al., 1992; Cook et al., 1994; Stevenson et al., 1994). Finally, examining the influence of mechanical loading on cortical defect healing in this model may help to discover other regulating factors between bone morphology and functional load.

\section{Discussion}

Knowledge of the basic biomechanics is essential throughout the care and management of patients with a long bone fracture. It starts from the evaluation of fracture mechanism to the selection of a treatment and immobilization method, followed by non-invasive assessment of healing and the evaluation of bone remodeling after the removal of the fixation device. Selection of a fixation method is multifactorial. A patient's age, the affected bone, fracture location and type, the existence of multiple trauma with extensive soft tissue involvement, and among many other factors, the skill, experience, and competence of a surgeon. However, the selection of a fixation device dictates the fracture-healing pathway governed by biological, mechanical and operative factors. Hence, the method of fracture immobilization can predispose the bone healing pathway and the appropriate course of patient management. This basic understanding is important not only for the consideration of bone fracture repair enhancement but also to prevent or minimize complications.

A full appreciation of the basic events in bone fracture healing following different pathways is essential for successful outcome of fracture treatment, regardless of the method of immobilization to be utilized. Hence, a good insight into the normal bone fracture healing mechanisms will be needed in the development of effective, reliable and practical interventions to further enhance the biological process which may already be optimal under normal circumstances. Although the underlying mechano-cellular interaction governing bone repair and remodeling are not completely understood, the basic science and clinical evidence are abundant to support the potential and prospect of biophysical stimulation to achieve Non-invasive Tissue Engineering in orthopaedic surgery and traumatology. However, before such possibility could become a reality, improved signal delivery transducers, effective method of monitoring tissue material and structural properties, and the establishment of a knowledge-based biophysical stimulation implementation strategy capable of optimizing the treatment to each patient's specific condition are of critical importance (Fig. 9).

According to Wolff's law, mechanical loading elicits an osteogenic response in bone structure. Controlled weight bearing under functional braces has a positive effect on tibial fracture healing (Sarmiento et al., 1984) and loading was proven to be a permissive factor for bone defect repair (Meadows et al., 1990). These and many other studies were successful in demonstrating the positive effect of physical stimuli at the structural and tissue level, but none was able to establish the mechanism for the cell biology-biomechanics interaction. The reason for this scientific void is par- tially related to the selection of appropriate experimental tools and partially due to the inherent technical difficulties facing the biologists, the practicing orthopaedic surgeons and bioengineers with widely different expectations concerning such technology. To make biophysical intervention effective in fracture healing and bone regenerate augmentation, a closer working relationship must be established among these disciplines under a full cooperation with the related device industry.

All the preliminary clinical and basic science results would strongly support the validity of biophysical stimulation. Unfortunately, each type of stimulus has countless combinations of treatment possibilities over a wide range of clinical conditions. The commercially available devices on the market lacks a strong commitment to demonstrate the fact that biophysical stimulation is as reliable and may even be more cost effective than other forms of intervention in managing similar problems in bone fracture and limb lengthening. Little effort has been devoted to optimize the dose effect by considering a time-related tissue change as the basis to adjust the stimulation signal intensity and treatment course. It would be highly desirable for the existing therapeutic delivery system to provide uniform stimulation field intensity at the focalized area of treatment with minimal scattering of physical energy to the adjacent normal tissue to prevent unexpected side effects. If the sciences and technology related to connective tissue modulation through biophysical stimuli are sufficiently advanced, why such treatment modality has not received the same acceptance in the clinical arena as compared to other equivalent biological or pharmacologic factors? Is it possible that such therapeutic modality happens to fall within the realm of Complementary and Alternative Medicine (CAM) thereby receiving much diluted attention or even unsubstantiated scepticism? Should one consider such non-invasive modality only as a prophylactic adjunct in difficult and complex cases? Or, perhaps the concept of biophysical stimulation is so simple and harmless thereby no supervision or regulation would be required in its application? These questions and arguments must be carefully deliberated not only to acquire equal acceptance of biophysical stimulation as a bonified medical intervention, but also to provide the appealing causes and rationale to drive the related science and technology forward.

It is important to realize that additional factors, both local and systemic, have been demonstrated to affect normal bone fracture healing under experimental conditions. Brighton (1984) concluded that, after an intensive review of literature, none of the studies involving the manipulation of biomechanical factors could show that the experimental fracture was able to reach the healed state more rapidly than the normal control without adverse conditions. He further emphasized that many factors may improve the early and mid-phases of fracture healing, but none seems able to shorten the time required for the healed bone to recover its normal mechanical strength. In a series of experiments conducted in our laboratory recently, the data appeared to corroborate with Brighton's original assertion. The same statement may apply to all forms of biophysical stimulation in that most of the enhancing effects seem to 

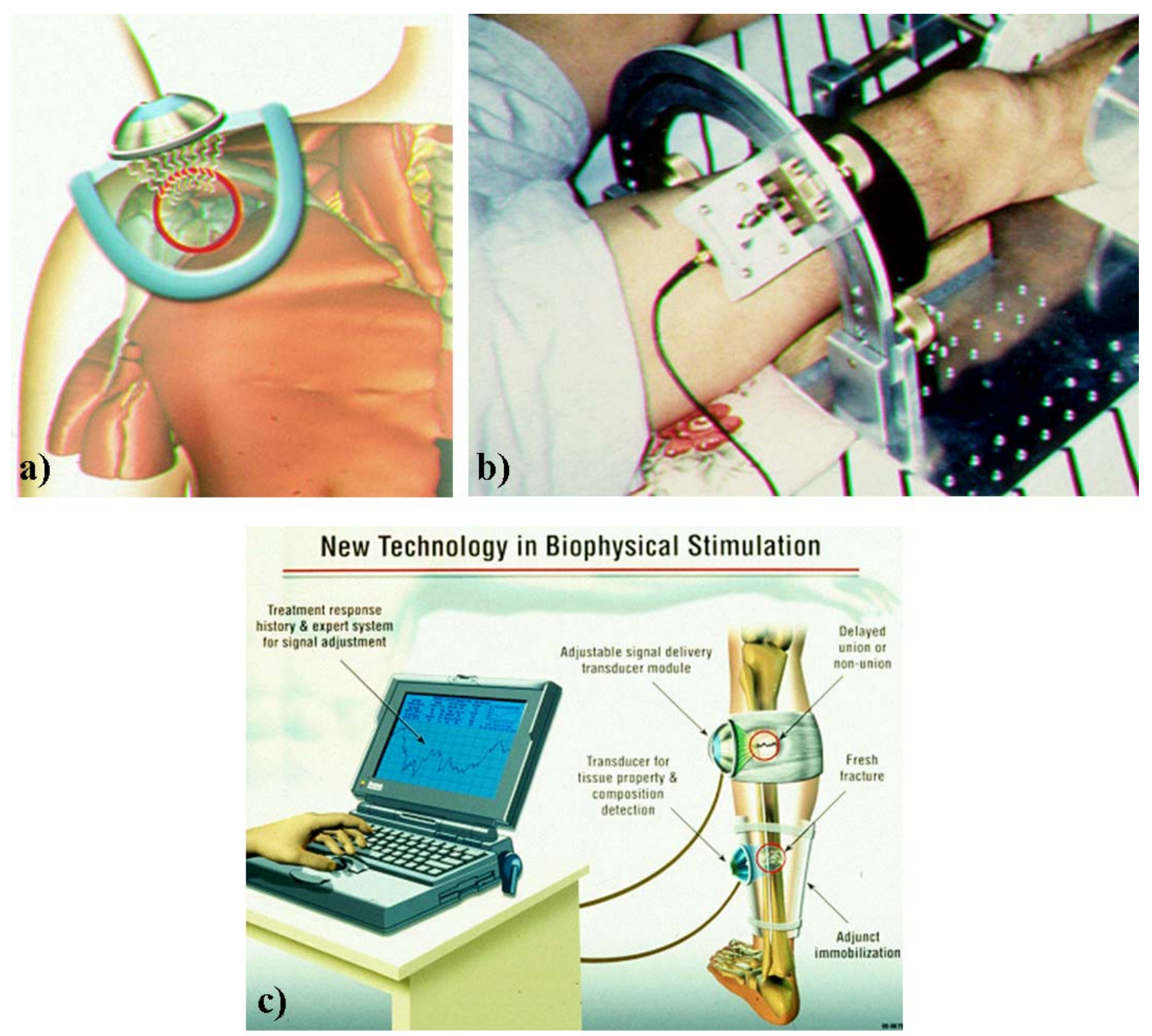

Figure 9. (a) Focalized stimulus delivery transducer with adjustable signal waveform and intensity for connective tissue repair and maintenance enhancement. (b) An ultrasonic device capable of quantifying bone material and structural properties. (c) The concept of knowledge-based biophysical stimulation implementation strategy individualized for each patient and the specific clinical problem involved.

exist only in early phases of fracture repair. This observation may relate to the fact that the late stage of bone remodeling is the longest period of fracture repair, which requires mechanical loading under the physiological frequency, magnitude and direction dictated by the musculoskeletal function. Such a process may not be entirely replaced by other means of enhancement without risking undesirable secondary effects. Therefore, the normal biologic response suggests that uncomplicated fracture healing is repairing at a rate that is near optimal. Enhancement of fracture healing, regardless of the type of intervention, would be indicated when the normal repairing mechanism is significantly compromised.

\section{Summary}

There is no doubt that biophysical interventions could provide effective augmentation to fracture healing. However, without knowing the precise cellular mechanisms associated with the associated osseous tissue transformation response under these interventions, it would be difficult and ineffective to implement appropriate therapy for the precise clinical indication. The time has come to combine the disciplines of biophysics and biomechanics with cell and molecular biology in a totally integrated fashion. Through such effort, the precise mechanism and enhancement effect on bone fracture repair, regeneration and remodeling under biophysical stimulation could be sufficiently quantified for clinical usage. Future research should include sig- 
nal transducer refinement, non-invasive monitoring of bone material and structural property changes, and the use of the knowledge-based treatment protocol to assure efficacy and reliability of this exciting and affordable therapeutic modality within the scientific realm of complementary and alternative medicine. Recognizing the potential difficulties in conducting experimental and clinical studies involving physicians, biologists, bioengineers and the technical personnel in the related industry with vastly different training and background, full awareness and appreciation of each field's limitations and unique attributes are essential to overcome the complex technical and non-technical problems encountered in all forms of biophysical stimulation. The end results of such seamless collaborative efforts should not be limited to bone fracture management alone. Positive enhancement factors could also benefit bone maintenance and regeneration, which affect many patients and the vast aging population worldwide.

\section{Acknowledgement}

This paper and the studies related to the discussion topic were supported in parts by a gift provided by EBI, LLP, Parsippeny, NJ and by a research grant funded by the AO Foundation, Davos, Switzerland.

\section{References}

Ament C, Hofer EP (2000) A fuzzy logic model of fracture healing. J Biomech 33: 961-968.

Aro HT, Chao EYS (1991) Biomechanics of fracture fixation. In: Basic Orthopaedic Biomechanics. Mow VC, Hayes WC (eds). Raven Press, New York, pp. 293-336.

Aro HT, Chao EYS (1993) Bone healing patterns affected by loading, fracture fragment stability, fracture type and fracture site compression. Clin Orthop 293: 8-17.

Aro HT, Wippermann BW, Hodgson SF, Wahner HW, Lewallen DG, Chao EYS (1989) Prediction of properties of fracture callus by measurement of mineral density using micro-bone densitometry. J Bone Joint Surg 71A: 10201030 .

Aro HT, Kelly PJ, Lewallen DG, Chao EYS (1990a) The effects of physiologic dynamic compression on bone healing under external fixation. Clin Orthop 256: 260-273.

Aro HT, Wippermann BW, Hodgson SF, Chao EYS (1990b) Internal remodeling of periosteal new bone during fracture healing. J Orthop Res 8: 238-246.

Aro HT, Wahner HT, Chao EYS (1991) Healing patterns of transverse and oblique osteotomies in the canine tibia under external fixation. J Orthop Trauma 5: 351-364.

Augat P, Margevicius K, Simon J, Wolf S, Suger G, Claes L (1998) Local tissue properties in bone healing: influence of size and stability of the osteotomy gap. J Orthop Res 16: 475-481.

Bassett CAL (1989) Fundamental and practical aspects of therapeutic uses of pulsed electromagnetic fields (PEMFs). Crit Rev Biomed Eng 17: 451-529.

Bassett CAL, Herrmann I. (1961) Influence of oxygen concentration and mechanical factors on differentiation of connective tissue in-vitro. Nature 190: 460-461.

Bodamyali T, Bhatt B, Hughes FJ, Winrow VR, Kanczler JM, Simmon B, Abbott J, Blake DR, Stevens CR (1998) PEMF simultaneously induce osteogenesis and upregulate transcription of BMP-2 and BMP-4 in rat osteoblasts in vitro. Biochem Biophys Res Commun 250: 458-461.

Bolander M (1998) Response of chondrocytes to low intensity ultrasound in culture. Workshop lecture, International Society for Fracture Repair, Biannual Meeting, Strasbourg. International Society for Fracture Repair (http:// fracture.com).

Borsalino G, Bagnacani M. Bettati E, Fornaciari F, Rocchi R, Uluhogian S, Ceccherelli G, Cadossi R, Traina GC (1988) Electrical stimulation of human femoral intertrochanteric osteotomies. Clin Orthop 237: 256-263.

Brighton CT (1984) Principles of fracture healing. In: Instructional Course Lectures, Vol. XXXIII. Murray JA(ed). C.V. Mosby, St. Louis. pp. 60-82.

Brighton CT, Hunt RM (1986) Ultrastructure of electrical induced osteogenesis in the rabbit medullary canal, J Orthop Res 4: 27-36.

Brighton CT, Black J, Friedenberg ZB, Esterhai JL, Day LJ, Connolly JF (1981) A multicenter study of the treatment of non-union with constant direct current. J Bone Joint Surg 63A: 2-13.

Brighton CT, Okereke E, Pollack S, Clark CC (1992) In vitro bone-cell response to a capacitively coupled electrical field: The role of field strength, pulse pattern, and duty cycle. Clin Orthop 285: 255-262.

Brighton CT, Wang W, Seldes R, Zhang G, Pollack SR (2001) Signal transaction in electrically stimulated bone cells. J Bone Joint Surg 83A: 1514-1523.

Buckwalter JA, Woo SLY, Goldberg VM, Hadley EC, Booth F, Oegema TR, Eyre DR (1993) Soft-tissue aging and musculoskeletal function. J Bone Joint Surg 75A: 1533-1548.

Carter DR (1987) Mechanical loading history and skeletal biology. J Biomech 20: 1095-1109.

Carter DR, Blenman PR, Beaupre GS (1988) Correlations between mechanical stress history and tissue differentiation in initial fracture healing. J Orthop Res 6: 736748.

Chao EYS, Aro H (1989) The effect of rigidity on fracture healing in external fixation. Clin Orthop 241: 24-35.

Chao EYS, Inoue N, Yoshida H, Toda K, Ito K, Schneider E (2004) Cortical defect repair and remodelling, a model to study the regulatory mechanism (abstr). Proceedings $50^{\text {th }}$ Orthopedic Research Society Meeting, San Francisco, CA. Trans Orthop Res Soc, in press.

Cheal EJ, Mansmann KA, DiGioia AM 3rd, Hayes WC, Perren SM (1991) Role of interfragmentary strain in fracture healing: ovine model of a healing osteotomy. J Orthop Res 9: 131-142.

Chow R, Harrison JE, Notarius C (1987) Effect of two randomized exercise programmes on bone mass of healthy postmenopausal women. Br Med J 292: 607-610.

Claes LE, Heigle CA (1996) The influence of local tissue strain on tissue differentiation and callus formation in 
secondary fracture healing (abstr). Proceedings of the Fifth Biennial Meeting of the International Society for Fracture Repair, Ottawa, Canada, p. 6. International Society for Fracture Repair (http://fracture.com).

Claes L, Augat P, Suger G, Wilke HJ (1997) Influence of size and stability of the osteotomy gap on the success of fracture healing. J Orthop Res 15: 577-584.

Claes LE, Wilke HJ, Kiefer H (1995) Osteonal structure better predicts tensile strength of healing bone than volume fraction. J Biomech 28: 1377-1390.

Cook SD, Baffes GC, Wolfe MW, Sampath TK, Rueger DC, Whitecloud TS III (1994) The effect of recombinant human osteogenic protein-1 on healing of large segmental bone defects. J Bone Joint Surg 76A: 827-838.

Cowin SC (1993) Bone stress adaptation models. J Biomech Eng 115: 528-533.

Cunningham NS, Paralkar V, Reddi AH (1992) Osteogenin and recombinant bone morphogenetic protein $2 \mathrm{~B}$ are chemotactic for human monocytes and stimulate transforming growth factor $\beta 1 \mathrm{mRNA}$ expression. Proc Natl Acad Sci USA 89: 11740-11744.

Delloye C, Dellefortrie G, Coutellier L, Vincent A (1990) Bone regenerate formation in cortical bone during distraction lengthening, an experimental study. Clin Orthop 250: 34-42.

Eickhoff JA, Molczyk L, Gallagher JC, De Jong S (1993) Influence of isotonic, isometric and isokinetic muscle strength on bone mineral density of the spine and femur in young women. Bone Miner. 20: 201-209.

Elias JJ, Frassica FJ, Chao EYS (2000) The open section effect in a long bone with a longitudinal defect - a theoretical modeling study. J Biomech 33: 1517-1522.

Eyres KS, Saleh M, Kanis JA (1996) Effect of pulsed electromagnetic fields on bone formation and bone loss during limb lengthening. Bone 18: 505-509.

Gallagher JC, Bishop CW, Knutson JC, Mazess RB, DeLuca HF (1994) Effects of increasing doses of 1hydroxyvitamin D2 on calcium homeostasis in postmenopausal women. J Bone Miner Res 9: 607-614.

Goodship AE, Kenwright J (1985) The influence of induced micromovement upon the healing of experimental tibial fractures. J Bone Joint Surg 67B: 650-655.

Hart RT (1990) A theoretical study of the influence of bone maturation rate on surface remodeling prediction: Idealized models. J Biomech 23: 241-253.

Hart RT, Davy DT, Heiple KG (1984) A computational method for stress analysis of adaptive elastic material with a view toward applications in strain-induced bone remodeling. J Biomech Eng 106: 342-350.

Heckman JD, Ryaby JP, McCabe J, Frey JJ, Kilcoyne RF (1994) Acceleration of tibial fracture-healing by noninvasive, low-intensity pulsed ultrasound. J Bone Joint Surg 76A: 26-34.

Huiskes R, Hollister SJ (1993) From structure to process, from organ to cell: Recent development of FE-analysis in orthopaedic biomechanics. J. Biomech Eng 115: 520 527.

Ilizarow GA (1989) The tension stress effect on the genesis and growth of tissue. Part II. The influence of the rate and frequency of distraction. Clin Orthop 239: 263285.
Inoue N, Toda I, Innis WP, Ito K, Chao EYS (2003) Time sequential changes in structural properties of a long bone with a longitudinal defect in a canine model. (abstr.). Proceedings of 49th Orthopaedic Research Society Meeting. Trans Orthop Res Soc 28: 427.

Inoue N, Ohnishi I, Chen DA, Deitz LW, Schwardt JD, Chao EYS (2002) Effect of pulsed electromagnetic fields on osteotomy gap healing in a canine tibial model. J Orthop Res 20: 957-966.

Kassis B, Glorion CH, Tabib W, Blanchard O, Pouliquen JC (1996) Callus response to micromovement after elongation in the rabbit. J Pediatric Orthop 16: 480-483.

Kristiansen TK, Ryaby JP, McCabe J, Frey JJ, Roe LR (1997) Accelerated healing of distal radius fractures with the use of specific, low intensity ultrasound. J Bone Joint Surg 79A: 961-973.

Kuiper JH, Richardson JB, Ashton BA (1996) Strains, hydrostatic pressures and fluid shear stresses in early fracture callus (abstr.). Proceedings of the Fifth Biennial Meeting of the International Society for Fracture Repair, Ottawa, Canada, p. 38. International Society for Fracture Repair (http://fracture.com).

Majeska RJ, Ryaby JT, Einhorn TA(1994) Direct modulation of osteoblastic activity with estrogen. J Bone Joint Surg 76A: 713-721.

Mammi GI, Rocchi R, Cadossi R, Massari L, Traina GC (1993) The electrical stimulation of tibial osteotomies. Clin Orthop 288: 246-253.

Markel MD, Wikenheiser MA, Chao EYS (1990) A study of fracture callus material properties: Relationship to the torsional strength of bone. J Orthop Res 8: 843-850.

Marsh JL, Nepola JV, Meffert RH (1992) Dynamic external fixation for stabilization of nonunions. Clin Orthop 278: $200-206$.

Meadows TH, Bronk JT, Chao EYS, Kelly PJ (1990) Effect of weight-bearing on healing of cortical defects in the canine tibia. J Bone Joint Surg 72A: 1074-1080.

Menkes A, Mazel S, Redmond RA, Koffler K, Libanati CR, Gundberg CM, Zizic TM, Hagberg JM, Pratley RE, Hurley BF (1993) Strength training increases regional bone mineral density and bone remodeling in middle-aged and older men. J Appl Physiol 74: 2478-2484.

O’Sullivan ME, Chao EYS, Kelly PJ (1989) Current concepts review. The effects of fixation on fracture healing. J. Bone Joint Surg 71A: 306-310.

Perren SM (1979) Physical and biological aspects of fracture healing with special reference to internal fixation. Clin Orthop 138: 175-196.

Rand JA, An KN, Chao EYS, Kelly PJ (1981) A comparison of the effect of open intramedullary nailing and comrpession-plate fixation on fracture site blood flow and future union. J. Bone Joint Surg 63A: 427-442.

Rubin CT, Lanyon LE (1984) Regulation of bone formation by applied dynamic loads. J Bone Joint Surg 66A: 397-402.

Rubin CT, Lanyon LE (1987) Osteoregulatory nature of mechanical stimuli: Function as a determinant for adaptive bone remodeling. J Orthop Res 5: 300-309.

Rubin C, Turner AS, Bain S, Mallinckrodt C, McLeod K (2001) Low mechanical signals strengthen long bones. Nature 412: 603-604. 
Rubin C, Turner AS, Muller R, Mittra E, McLeod K, Lin W, Qin YX (2002) Quantity and quality of trabecular bone in the femur are enhanced by a strongly anabolic, noninvasive mechanical intervention. J Bone Mineral Res 17: 349-357.

Ryaby JT (1998) Clinical effects of electromagnetic and electric fields on fracture healing. Clin Orthop 355 Suppl: S205-215.

Sarmiento A, Sobol PA, Hoy AL, Ross SDK, Racette WL, Tarr RR (1984) Prefabricated functional braces for the treatment of fractures of the tibial diaphysis. J Bone Joint Surg 66A: 1328-1339.

Sato W, Matsushita T, Nakamura K (1999) Acceleration of increase in bone mineral content by low-intensity ultrasound energy in leg lengthening. J Ultrasound Med 18: 699-702.

Schaden W, Fischer A, Sailler A (2001) Extracorporeal shock wave therapy of nonunion or delayed osseous union. Clin Orthop 387: 90-94.

Sharrard WJW (1990) A double-blind trial of pulsed electromagnetic fields for delayed union of tibial fractures. J Bone Joint Surg 72B: 347-355.

Srinivasan S, Weimer DA, Agans SC, Bain SD, Gross TS (2002) Low-magnitude mechanical loading becomes osteogenic when rest is inserted between each load cycle. J Bone Min Res 17: 1613-1620.

Stevenson S, Cunningham NS, Davy D, Reddi AH (1994) The effect of osteogenin (a bone morphogenetic protein) on the formation of bone in orthotopic segmental defects in rats. J Bone Joint Surg 76A: 1676-1687.

Tanaka SM, Alam IM, Turner CH (2003) Stochastic resonance in osteogenic response to mechanical loading. FASEB J 17: 313-314.

Taylor KF, Inoue N, Rafiee B, Tis JE, McHale KA, Chao EYS (2003) The effect of pulsed electromagnetic fields on regenerate bone in a rabbit limb lengthening model(abstr.). Proceedings of the $49^{\text {th }}$ Orthopedic Research Society Meeting, New Orleans, LA. Trans Orthop Res Soc 28: p. 067

Tis JE, Meffert RH, Inoue N, McCarthy EF, Machen MS, McHale KA, Chao EYS (2002) The effect of low intensity pulsed ultrasound applied to rabbit tibiae during the consolidation phase of distraction osteogenesis. J Orthop Res 20: 793-800.

Wang CJ, Huang HY, Chen HH, Pai CH, Yang KD (2001) Effect of shock wave therapy on acute fractures o the tibia. Clin Orthop 387: 112-118.

Windhager R, Tsuboyama T, Siegl H,Groszschmidt K, Seidel G, Schneider B, Plenk H Jr (1995) Effect of bone cylinder length on distraction osteogenesis in the rabbit tibia, J Orthop Res 13: 620-628.

Wolff, J (1986) The Law of Bone Remodeling. Springer, Berlin.

Wu JJ, Shyr HS, Chao EYS, Kelly PJ (1984) Comparison of osteotomy healing under external fixation devices with different stiffness characteristics. J. Bone Joint Surg 66A: 1258-1264.

Xavier CAM, Duarte LR (1983) Estimulaci ultra-sonica de callo osseo: Applicaca clinica (Ultrasonic investigation of bone callus. Clinical application). Rev Brasiliera Orthop 18: $73-80$.
Yang KY, Parvizi J, Wang SJ, Lewallen DG, Kinnick RR, Greenleaf JF (1996) Exposure to low intensity ultrasound increases aggregan gene expression in a rat femur fracture model. J Orthop Res 14: 802-809.

Yasuda I (1955) Dynamic callus and electric callus. J Bone Joint Surg 37A:1292-1299.

Yasui N, Kojimoto H, Sasaki K, Kitada A, Shimizu H, Shimomura Y (1993) Factors affecting callus distraction in limb lengthening, Clin Orthop 293: 55-60.

\section{Discussion with Reviewers}

U. Ripamonti: Do you feel that elucidating the intracellular events initiating and mediating tissue repair and remodelling can be most effectively studied in an animal model where there are a plethora of confounding variables. Is it not more feasible to test a hypothesis invitro before testing it in vivo?

Authors: Intracellular responses due to biophysical stimulation would not be effectively studied in an animal model. Such effects may only be investigated and quantified using an in vitro cell or tissue culture setup. However, in order to establish the phenomena of biophysical stimulation on connective tissue augmentation, one must utilize an animal model first before relying on the in vitro models to establish the molecular mechanism and proceed to work on the optimal signal waveform and dose. I have elaborated my opinions about this in the "Animal Model Selection" section of this paper.

U. Ripamonti: You suggest that if the cellular responses to physical interventions could be identified, bone healing could ultimately be induced by a chemical or pharmaceutical agent, replacing physical modulation. And indeed this is an active area of research by investigators using morphogens such as BMPs and TGF- s. Did you not feel that this facet of bone healing had a place in the review?

Authors: Yes, definitely! However, we are not qualified to do that and this important aspect on bone formation and repair stimulation falls outside of the main scope of the present review paper.

S.Fenwick: In the light of some very good clinical evidence for certain non-invasive devices, what do you think needs to be done to convince the clinical and scientific community as a whole that non-invasive stimulation is a viable treatment modality?

Authors: This is an extremely important question! We have tried to speculate the possible answers to this question in the "Discussion" section of this paper. Briefly, we suggested that we need to make such intervention a "Prescription" treatment similar to the prescription drugs or the prescription-based imaging modalities prescribed and reviewed only by qualified physicians while the stimulation treatment could be carried out by trained and certified technicians.

S. Fenwick: Would you like to speculate as to how good some of these potential therapies could actually be?

Authors: Another important question! We have coined the 
term "Non-invasive Tissue Engineering" to express our enthusiasm towards this technology as a safe, effective, reliable and affordable therapeutic modality. However, we need NASA (not NIH) type of expertise and funding in making it a reality! In addition, this may only occur with the strong endorsement, utilization and enthusiasm from the practicing physicians.

S. Fenwick: There is a lot of belief that these technologies are not in fact efficacious, partly because there are so many devices claiming so many effects. How do we go about separating the 'good' technologies from the 'bad' technologies?
Authors: This monumental obstacle could only be overcome if such technology were under the usual medical practice protection. The related concept and devices involved in different forms of biophysical stimulation are too easy to copy and being applied without appropriate prescription and supervision, which would severely reduce the effectiveness of such therapy and thus confusing and misinform the public. Worse yet, utilizing such technology in an indiscriminative manner may cause undesirable and even dangerous secondary effect. Hence, making it a "Prescription" treatment based only on scientific data, knowledge and outcome evidence is the only way to solidify its position and trust in the medical community and among the lay public. 